\title{
Electronic Properties of Organic Semiconductor/Electrode Interfaces: The Influence of Contact Contaminations on the Interface Energetic
}

\author{
M. Grobosch* and M. Knupfer
}

IFW Dresden, P.O. Box 270116, D-01171 Dresden, Germany

\begin{abstract}
During the last decades there has been considerable progress as regards the understanding of OSC/electrode interfaces. In this Review, we summarize recent work on the interface energetics influenced by the presence of electrode surface contamination due to the used ex-situ cleaning procedure prior the interface formation. Contact contaminations of the electrodes essentially affect the interface energetics in particular the interface parameters and therefore the performance of organic electronic devices. These new insights in the interface energetics at so-called realistic OSC/metal interfaces can help in the future to better design and optimize organic electronic devices such as OLEDs, OFETS, and OPVs. The reviewed investigations of the interface energeticss of interfaces relevant for organic spintronic devices, and in particular the effect of contamination layer induced changes in the energy level alignment of such interfaces lead to a significant progress in the understanding of the interfacial properties of organic spintronic relevant interfaces. They represent one step toward the description of spin transport in organic spintronic devices.
\end{abstract}

Keywords: Photoemission spectroscopy, contamination layer, interfaces, organic semiconductor.

\section{INTRODUCTION}

In the last decades organic semiconductors (OSC) have demonstrated their great potential for applications in organic electronic devices [1-3] such as organic light emitting diodes (OLEDs) [4-8], organic thin film transistors (OTFTs) [9-12], and organic photovoltaic devices (OPVs) [13-15]. At this the understanding of the interface characteristics in organic electronic devices is of crucial importance for the further advance in the device performance and the device design. Experimental and theoretical investigations of the interfaces and the surfaces involved in organic electronic devices have generated critical insight of the fundamental processes at interfaces between organic semiconductors and the electrode material as well as OSC heterojunctions. Considerable experimental and theoretical work has been devoted to the topic of OSC/electrode interfaces, and detailed reviews and monographs of general results as well as interpretations have been published [1, 3, 7, 16-27].

While the main work has been focussed on the energy level alignment between OSCs and under ultra-high vacuum (UHV) conditions prepared, atomically clean substrate surfaces, over the past decades and after some detailed organic/metal interface investigations [28-34] it has been transpired that contact contaminations play an important role at OSC/electrode interfaces and for the corresponding interface energetics. In practise organic electronic devices will be hardly fabricated under high vacuum and UHV, respectively. Often the device fabrication involves the processing of the

*Address correspondence to this author at the IFW Dresden, P.O. Box 270116, D-01171 Dresden, Germany; Tel: +49-(0)351-4659-409; Fax: +49(0)351-4659-313; E-mail: m.grobosch@ifw-dresden.de electrode surfaces in controlled atmospheres (e.g. in a glove box, $\mathrm{N}_{2}$-atmosphere or under poor vacuum conditions) and under ambient conditions. Under that condition the molecules of atmosphere $\left(\mathrm{N}_{2}, \mathrm{H}_{2} \mathrm{O}, \mathrm{CO}, \mathrm{O}_{2}\right.$, and hydrocarbons) adsorb on the electrode surface.

Due to the adsorbed molecules a contamination layer on top of the electrode surface, a partial oxidation, and/or the passivation of the electrode surface can be the consequence. It is therefore important to review the differences in the interface energetics that have been demonstrated for interfaces involving contaminated electrode surfaces with respect to interfaces of OSCs in contact to atomically clean substrate surfaces formed under UHV conditions. The purpose of this review is therefore not to review the considerable body of experimental and theoretical knowledge published on OSC/electrode interfaces. Within the frame work of this review the focus will be (i) on the influence of contact contaminations on the energy level alignment at OSC/metal interfaces and (ii) on interfaces relevant for organic spintronic devices, and in particular on the effect of contamination layer induced changes in the interface energetics of such interfaces.

\section{EXPERIMENTAL ASPECTS: PHOTOEMISSION SPECTROSCOPY}

Most of the experimental publications that have been reported in the past decades regarding the energy level alignment at OSC/electrode interfaces have been obtained by means of photoemission spectroscopy (PES). This experimental technique is a powerful tool for the investigation of the chemistry and electronic properties of interfaces and surfaces because of its high surface sensitivity due to the very small escape depth of the photoelectrons and the fact that 
PES is a non-destructive method for most OSCs. PES is the most important experimental method to determine band offsets at OSC/electrode interfaces as well as OSC heterojunctions. The technique itself as well as the use of PES for the investigation of OSC/electrode interfaces has been considered in great detail before in many monographs and review articles see for instance Refs. [16-18, 21, 24, 26, 27, 35-38].

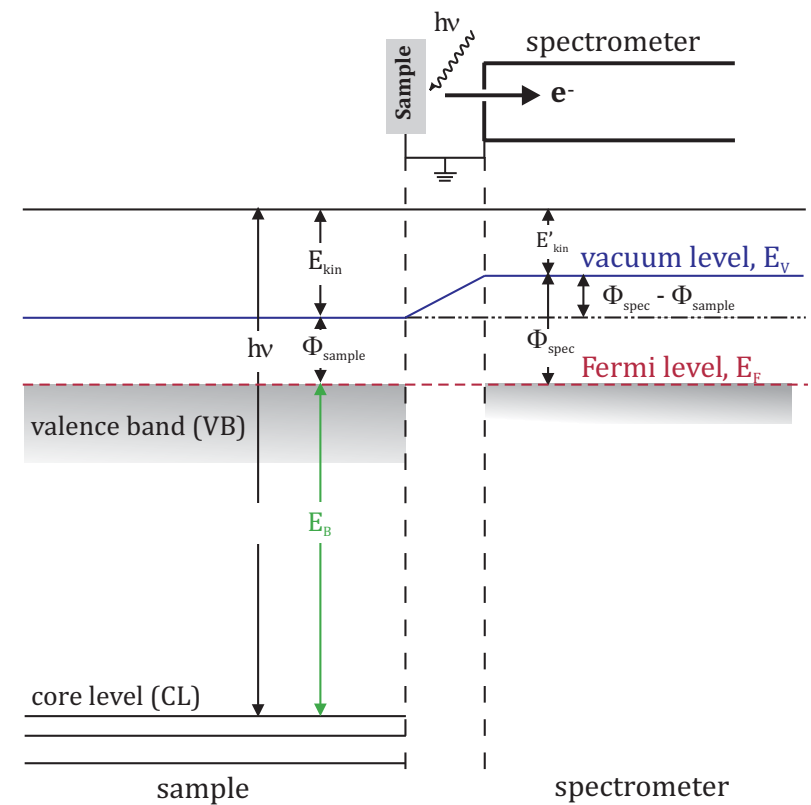

Fig. (1). Schematic representation of the photoemission process for the sample and the spectrometer in a photoemission spectroscopy experiment (From Ref. [24, 39]).

Photoemission spectroscopy represents an experimental technique, which is based on the photoelectric effect, probing the occupied states of a given system. In this kind of experiment electrons, so-called photoelectrons, from occupied states are excited above the vacuum level $\left(\mathrm{E}_{\mathrm{V}}\right)$ and can thus escape the sample. In a PES experiment the sample is irradiated by monochromatic light with a photon energy hv under UHV conditions with a pressure in the range of $10^{-10}$ mbar and photoelectrons are emitted. In this way it is possible to perform core level spectroscopy when a core level electron is excited and valence band spectroscopy by exciting valence band electrons, respectively (see Fig. 1, left hand side). At this, X-rays are most commonly used to excite high binding energy core level electrons (X-ray photoemission spectroscopy, XPS) and He discharge lamps providing vacuum ultraviolet radiation to emit electrons from the valence band region (Ultraviolet photoemission spectroscopy, UPS). In a first approximation, the measured kinetic energy $\left(\mathrm{E}_{\mathrm{Kin}}\right)$ of the excited photoelectron allows the determination of its binding energy (EB) via the simple equation:

$$
\mathrm{E}_{\mathrm{Kin}}=\mathrm{hv}-\mathrm{E}_{\mathrm{B}}-\Phi_{\text {spec }}
$$

where hv displays the photon energy and $\Phi_{\text {spec }}$ is a spectrometer specific constant, the work function of the spectrometer. Only photoelectrons whose kinetic energy is higher than the work function $\Phi_{\text {sample }}$ of the sample can escape from the surface. Fig. (1) illustrates this effect. In a real PES experiment the photoelectron has to enter the analyzer where its kinetic energy $\mathrm{E}_{\mathrm{Kin}}$ is measured. Therefore, it has to over- come the contact potential $\Phi_{\text {spec }}-\Phi_{\text {sample }}$ as depicted on the right hand side of Fig. (1). As displayed in Fig. (1) the Fermi levels of the sample and the spectrometer are aligned in thermodynamic equilibrium. If one subtracts $\left(\Phi_{\text {spec }}-\Phi_{\text {sample }}\right)$ from Eq. 1 the formula remains the same but with $\Phi_{\text {spec }}$ in-

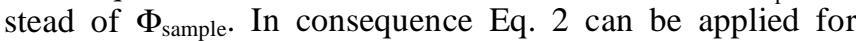
$\mathrm{E}_{\text {Kin }}^{\prime}$ :

$$
\mathrm{E}_{\mathrm{Kin}}^{\prime}=\mathrm{h} v-\mathrm{E}_{\mathrm{B}}-\Phi_{\text {spec }}=\mathrm{E}_{\mathrm{Kin}}-\left(\Phi_{\text {spec }}-\Phi_{\text {sample }}\right)
$$

Taking into account $\Phi_{\text {spec }}$ as a constant for the experimental setup the binding energy EB of electronic states of any conductive sample can be monitored regardless of $\Phi_{\text {sample. }}$ Simply speaking, the distribution of kinetic energies of the excited photoelectrons corresponds directly to the electronic density of states in the sample.

As described above, only photoelectrons with a $E_{\text {Kin }}$ higher than the work function of the sample can escape from the surface. In consequence, the work function of the sample can be determined by using UPS. Typical UPS spectra for a metallic substrate (top curve, example: Ag) and for OSC (bottom curve, example: OSC $\alpha-6 \mathrm{~T}$ ) are depicted in Fig. (2). Two energies are definitively responsible for the width $\mathrm{w}$ and the energy position of the spectrum. The first energy, the so-called Fermi energy $E_{F}=E_{B}=0 \mathrm{eV}$ of the metal substrate defines the zero position of the binding energy axis. The Fermi energy is characterized by electrons, which escape from the sample with the lowest binding energy (highest $\left.E_{\text {Kin }}\right)$. The width $w$ of the UPS-spectrum results from the energy difference between the Fermi energy and the high binding energy electron cutoff (HBEC). The energy position of the HBEC corresponds to photoelectrons, which are barely able to leave the sample with $\mathrm{E}_{\mathrm{Kin}} \cong 0 \mathrm{eV}$. These secondary electrons stem from within the sample scattered electrons. As visible from Fig. (1) in a real PES experiment all photoelectrons with a kinetic energy in the range $\mathrm{E}_{\text {Kin }}<\left(\Phi_{\text {spec }}-\Phi_{\text {sample }}\right)$ cannot be monitored. In order to overcome this potential barrier and to distinguish between the analyzer and the sample HBEC a bias voltage of a few volts (typically 3-9 eV) has to be applied to the sample.

The procedure to determine the features such as the energy position of the HBEC, of the hole injection barrier as well as of the Fermi level is illustrated in Fig. (3). The position of the HBEC is defined as the highest binding energy of the monitored photoelectrons. Its energy position can be determined by linear extrapolation to the binding energy axis (ref. Fig. (3) panel (a)). The energy position of the highest occupied molecular orbital (HOMO) of the OSC is generally referred by its low energy onset. This onset can be used to determine the barrier for hole injection $\Phi_{\mathrm{HIB}}$ by performing a linear extrapolation of the low binding energy edge of the HOMO feature as shown in Fig. (3) in panel (b). The determination of the Fermi level of a metal differs from the determination of the UPS feature position including the position of the HBEC and the HOMO onset. They can be realized following Fig. (3) panel (c). For a metal the measured Fermi level is expected to obey Fermi statistics. Therefore, the Fermi level is defined to occur as the point where the energy level is half occupied, i.e., the midpoint of the leading 


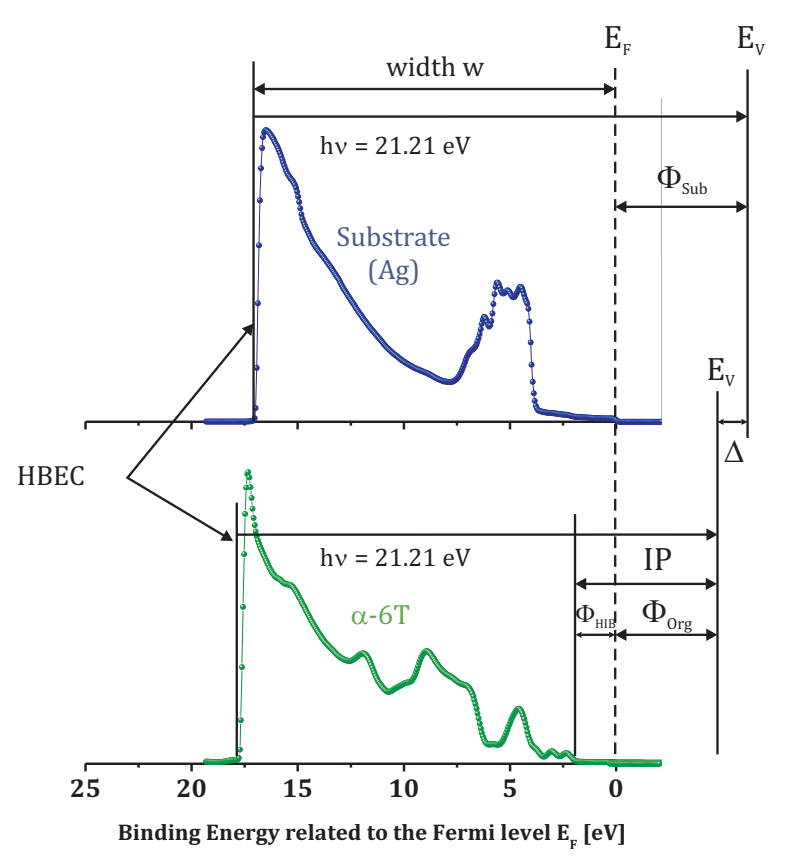

Fig. (2). Schematic determination of the interface parameters at OSC/electrode interfaces using ultraviolet photoemission spectroscopy. Example: $\alpha-6 \mathrm{~T} / \mathrm{Ag}$ interface (Adopted from Ref. [21, 40]).
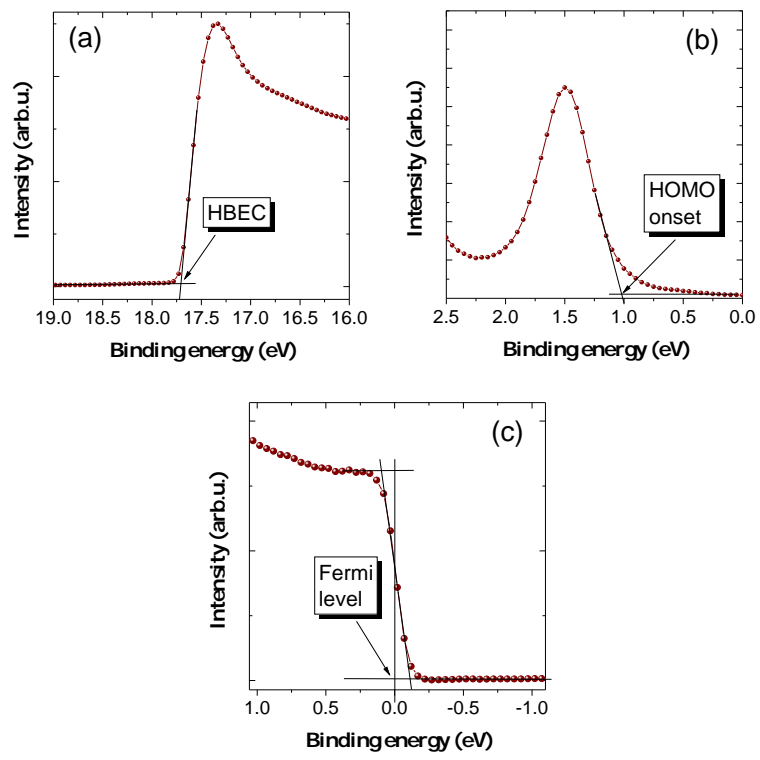

Fig. (3). Determination of the features of an UPS spectra, including the high binding energy cutoff (HBEC) (panel (a)), the onset of the highest molecular orbital (HOMO) of an OSC (panel (b)), and the position of the Fermi level of a metal (panel (c)) (After Ref. [27, 40]).

edge of the spectrum, as depicted in Fig. (3) panel (c). The relevant parameters in the context of OSC/electrode interfaces such as the substrate and the OSC work function $\Phi_{\text {Sub }}$ and $\Phi_{\text {Org }}$, the interface dipole $\Delta$, the hole injection barrier $\Phi_{\text {HIB }}$ as well as the ionization potential of the organic semiconductor IP can be easily estimated from the UPS spectra as illustrated in Fig. (2). In correspondence to Fig. (2) the relations to determine the interface parameters $\Phi_{\text {Sub }}$ and $\Phi_{\text {Org }}, \Delta$, $\Phi_{\mathrm{HIB}}$, and IP are

$$
\begin{aligned}
& \Phi_{\text {Sub }}, \Phi_{\text {Org }}=\mathrm{h} v-\mathrm{w}=\mathrm{h} v-\mathrm{E}_{\mathrm{B}, \mathrm{HBEC}}-\mathrm{E}_{\mathrm{F}} \\
& \Delta=\mathrm{HBEC}_{\mathrm{Org}}-\mathrm{HBEC}_{\mathrm{Sub}} \\
& \Phi_{\mathrm{HIB}}=\mathrm{E}_{\mathrm{B}, \mathrm{HOMO}}-\mathrm{E}_{\mathrm{F}} \\
& \mathrm{IP}=\Phi_{\text {Org }}+\Phi_{\mathrm{HIB}} .
\end{aligned}
$$

The reviewed experiments [107] have been carried out using a commercial PHI 5600 spectrometer, which is equipped with two light sources providing photons with energy of $1486.6 \mathrm{eV}$ from a monochromatized $\mathrm{Al} \mathrm{K} \alpha$ source for XPS and photons from a Helium lamp with energy of $21.21 \mathrm{eV}$ for UPS experiments. All UPS measurements have been done by applying a sample bias of $-5 \mathrm{~V}$ to obtain the correct, sample determined, HBEC.

The recorded spectra were corrected for the contributions of He satellite radiation. The total energy resolution of the spectrometer was determined by analyzing the width of the Fermi edge of a metallic substrate (for example: Au, Pd or $\mathrm{Ag}$ ) to be about $350 \mathrm{meV}$ (XPS) and $100 \mathrm{meV}$ (UPS), respectively. The binding energy scale was aligned by measuring the Fermi edge and the respective metallic substrate emission feature (for example: Au $4 \mathrm{f}_{7 / 2}$ ). In a preparation chamber (base pressure: $1-2 \cdot 10^{-10} \mathrm{mbar}$ ), which is directly attached to the spectrometer system thin films of the used organic semiconductors with different film thicknesses have been prepared by in-situ thermal evaporation with a typical evaporation rate between $1 \AA / \mathrm{min}$. and $2.5 \AA / \mathrm{min}$. on the respective substrate. Subsequently, the films were transferred to the analyzer chamber (base pressure: $1 \cdot 10^{-10}$ ) without breaking the vacuum and characterized taking a full range XPS spectrum. The number of impurities in the films was very small and below the detection limits of the XPS due to the UHV conditions during the preparation process of the organic films. To estimate the thickness of every individual organic overlayer we have monitored the attenuation of the intensity of the respective substrate peak due to the organic film. Considering the procedure of Seah and Dench [41] we have calculated the mean free path of the electron in the different organic materials. We point out that this procedure to determine the thickness of the organic layers is only correct for a layer-by-layer growth. If the organic films does not grow uniformly this method underestimates the film thickness. The thickness of the contamination layer on ex-situ treated substrate surfaces were estimated by analyzing the attenuation of the intensity of the characteristic substrate peak of an in-situ cleaned substrate in comparison to the characteristic substrate peak of a contaminated substrate. The quantification of the thickness was carried out using the mean free path according to Seah and Dench [41]. We estimate the error of this thickness determination to be $0.5 \mathrm{~nm}$. In Fig. (6 \& 7), and Fig. (9) we have additionally included the LUMO (lowest unoccupied molecular orbital) position which reflects the barrier for electron injection. The LUMO position has has been obtained taking into account the exciton binding energy $\mathrm{E}_{\mathrm{b}}$ in $\mathrm{CuPc}$ (copper(II)-phthalocyanine) of $0.6 \mathrm{eV}$ and in $\alpha-6 \mathrm{~T}$ ( $\alpha$-sexithiophene) of $0.4 \mathrm{eV}$ [42] and the onset of the electron-hole excitation $\mathrm{E}_{\mathrm{eh}}$ of about $1.5 \mathrm{eV}$ $(\mathrm{CuPc})$ [43] and $2.2 \mathrm{eV}(\alpha-6 \mathrm{~T})$ [44]. These values provide 
the band gap $E_{g}=E_{b}+E_{e h}$ and thus the energy distance of the HOMO and LUMO onsets.

\section{INFLUENCE OF CONTACT CONTAMINATIONS ON THE ENERGY LEVEL ALIGNMENT OF OSC/ METAL INTERFACES}

Already in 1995 S. Narioka et al. [28] have published a work on the influence of oxygen on the injection barriers at interfaces between the OSC ZnTPP (5,10,15,20-zinctetraphenylporphyrin) and various metals $(\mathrm{Au}, \mathrm{Ag}, \mathrm{Al}, \mathrm{Mg}$ ) using ultraviolet photoemission spectroscopy. The under UHV conditions prepared interfaces shows a similarity of the spectral shape indicating the absence of changes in the electronic structure of ZnTPP in contact to the different metal substrates. Contrary to the spectral shape the peak position depends significantly on the metal substrate. For all four interfaces the electronic energy levels of the OSC ZnTPP align to the vacuum level of the metal substrate. At this, a constant energy shift of the vacuum level across the interfaces could be observed. After the exposure to $1 \cdot 10^{-9} \mathrm{~L}$ oxygen $(\mathrm{p}=4$ Torr, $\mathrm{t}=5 \mathrm{~min}$ ) the shape of the spectral features show no significant change compared to that in UHV measurements. This suggests weak interaction between ZnTPP and oxygen. Due to the exposure to oxygen the valence band structures of ZnTPP are shifted relative to the Fermi level of the metal substrates with a different extend and direction depending on the metal substrate. After the exposure to oxygen of the ZnTPP/metal systems a shift of the energy levels in close relation with the change of the metal substrate work function by $\mathrm{O} 2$ have been found.

C. Shen and co-workers [29] addressed the effect of an oxid layer on the electrode surface on the example of a $\mathrm{Mg}: \mathrm{Ag} / \mathrm{Alq}_{3} / \mathrm{Mg}: \mathrm{Ag}$ structure in $1999 . \mathrm{Mg}: \mathrm{Ag} / \mathrm{Alq}_{3} / \mathrm{Mg}: \mathrm{Ag}$ were fabricated and characterized under UHV conditions using X-ray photoemission spectroscopy and I-V measurements, with and without exposure of the bottom electrode to an atmosphere of nitrogen including a substantial amount of oxygen and water vapour. Prior to the exposure of the bottom contact to the atmosphere no trace of oxygen could be detected and the $\operatorname{Mg} 2 p$ and $\mathrm{Mg} 2 s$ core level spectra, respectively, exhibits only structures which are characteristic for metallic magnesium. After the exposure a large $\mathrm{O} 1 s$ doublet is observed as an indication for the formation of magnesium hydroxide and magnesium oxid. Also the core level excitation of the $\mathrm{Mg}$ substrate underlines the formation of a magnesium oxide layer with an estimated thickness of $1.5 \pm 0.5$ $\mathrm{nm}$. The additionally performed I-V measurements show for the structures prepared under UHV conditions a symmetric I$\mathrm{V}$ characteristic compared to a clear asymmetric behavior due to the formation of a $\mathrm{Mg}$ oxide layer on the bottom electrode following ambient exposure.

A. Kahn and co-workers used UPS and I-V measurements to investigate the injection barrier for hole and the injection characteristic for OSC in contact to clean and contaminated electrode surfaces as depicted in Fig. (4 and $\mathbf{5})$ for the OSC $\alpha$-NPD (N,N'-diphenyl-N,N'-bis(1-naphthyl)-1,1'biphenyl-4,4'-diamine) [30] and $\mathrm{Alq}_{3}[26,31]$ in contact to $\mathrm{Au}$, with and without the exposure to ambient conditions. For both systems the contaminated Au electrode surface was prepared by exposure of an atomically clean, under UHV

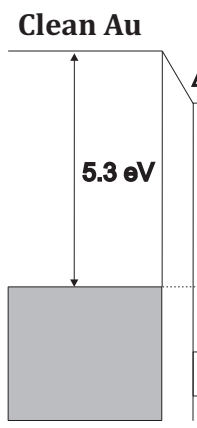

(a)

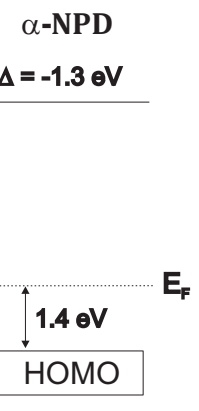
Fig. (4). Interface energeticss for (a) $\alpha-N P D / c l e a n ~ A u$ and (b) $\alpha$ NPD /contaminated Au, as determined by UPS. With Cont. the on the surface present contamination layer due to the ex-situ treatment of the electrode surface is denoted (Adopted from Ref. 30).

conditions prepared Au surface for a few minutes to ambient conditions. Performed AES (Auger electron spectroscopy) measurements reveal a contamination layer of carbon oxygen with a thickness of 0.5-1 monolayer. Furthermore, no sign of any contamination is detected on the clean Au substrate surface. As a consequence the work function of the exposed Au surface $(\Phi=4.7-5.0 \mathrm{eV})$ is strongly reduced with respect to that of the in-situ prepared, clean electrode surface $\Phi=5.3-5.4 \mathrm{eV}$. Additionally significant variations of the work function of contaminated Au surfaces have been observed ranging from $4.7 \mathrm{eV}$ to $5.1 \mathrm{eV}$, whereas the work function of the in-situ prepared Au surfaces vary only between $5.3 \mathrm{eV}$ and $5.4 \mathrm{eV}$. The UPS measurements show that the barrier for hole injection is reduced for the interfaces with the contaminated low work function Au substrate in comparison to the clean, high work function Au electrode. The additionally performed I-V measurements confirm this unexpected result for the barriers [26, 30].

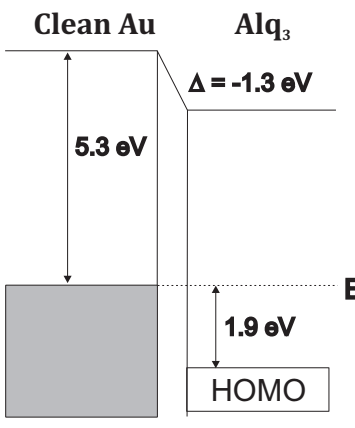

(a)

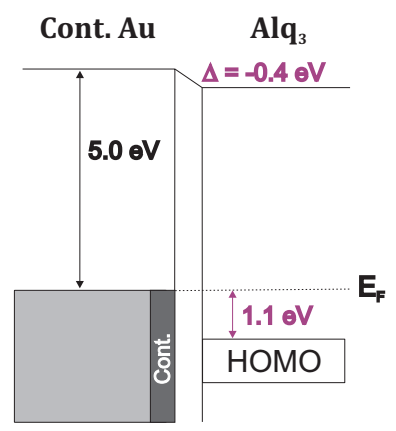

(b)
Fig. (5). Energy level alignment of interfaces of thin vacuum evaporated $\mathrm{Alq}_{3}$ films and $\mathrm{Au}$. On the left hand side (panel (a)) the interface energeticss for the interface $\mathrm{Alq}_{3} /$ clean $\mathrm{Au}$ and on the right hand side (panel (b)) for the interface Alq3/contaminated $\mathrm{Au}$ is depicted, as determined using UPS. With "Cont." the on the surface present contamination layer due to the ex-situ treatment of the electrode surface is denoted (Adopted from Ref. [26, 31]).

In order to explore and determine the effect of contact contaminations on the energetics of realistic OSC/metal interfaces in more detail, we carried out a systematic combined $\mathrm{X}$-ray and ultraviolet photoemission spectroscopy study of interfaces between the OSC $\alpha-6 \mathrm{~T}$ and various polycrystalline metal substrates ( $\mathrm{Au}, \mathrm{Ag}, \mathrm{Pd}$, and $\mathrm{Pt})$, whereby the metal work function changes by more than $1 \mathrm{eV}[32,45,46]$. Due 
to the wide fact, that thiophene-based OSC are widely used in the preparation of organic electronic devices (e.g. OFETs [9-11], organic spintronic devises $[47,48]) \alpha-6 \mathrm{~T}$ is a good representative of this material class. In order to obtain atomically clean (ideal) metal surfaces the polycrystalline substrates were in-situ cleaned by several cycles of $\mathrm{Ar}^{+}-$ sputtering [21, 49]. Prior to the in-situ cleaning the metal substrates was rinsed with ethanol. To probe contaminated (realistic) metal contacts, we have treated the polycrystalline metal substrates in an ultrasonic bath with acetone for 15 min. and additional rinsing with ethanol for $1 \mathrm{~min}$. before putting the substrates into the UHV chamber. XPS measurements of ex-situ cleaned substrates reveal obviously a contamination layer on top of the substrate surfaces consisting of carbon (75\%), oxygen (20\%), and nitrogen $(5 \%)$. The thickness of the contamination is estimated to be about 1-2 $\mathrm{nm}$. Whereas the in-situ cleaning of the substrate results in a contamination free, atomically clean surface. Significant differences can be also observed in the UPS spectra for exsitu and in-situ cleaned substrates. For all ex-situ cleaned metal substrates a featureless valence band and a significant decrease in the work function with respect to that of the atomically clean surfaces were observed due to the present contamination layer. The investigated interfaces between $\alpha$ 6T and in-situ cleaned $\mathrm{Au}, \mathrm{Ag}, \mathrm{Pd}$, and Pt substrates are illustrated in Fig. (6). They all show a substantial hole injection barrier from $0.6 \mathrm{eV}$ for the $\alpha-6 \mathrm{~T} / \mathrm{Pd}$ system up to 1.8 $\mathrm{eV}$ for the 6T/Ag interface. Furthermore, the interfaces are characterized by a large interface dipole $-0.7 \mathrm{eV}$ to $-1.5 \mathrm{eV}$. In the literature several contributions to the interface dipole are discussed $[16,17,21,22,50,51]$. The interface dipole at OSC/metal interfaces can be attributed for example to: (i) a reduction of the metal work function upon the adsorption of the OSC molecules19, [52-56], (ii) chemical interactions between the OSC molecules and the metal surface, and (iii) an induced density of states, which defines the charge neutrality level (CNL) of the OSC, while there is a tendency to align the Fermi level of the metal and the CNL of the OSC. In particular, for the $\alpha-6 \mathrm{~T} / \mathrm{Ag}$ interface [45] the additional feature in the former energy gap of OSC at $1.4 \mathrm{eV}$ binding energy suggest the formation of an interface related electronic level due to chemical interaction, a negative charge transfer from silver to the OSC. These interface states can pin the interface Fermi level and in this way predominantly define the interface energeticss. For the interface $\alpha-6 \mathrm{~T}$ $6 \mathrm{~T} / \mathrm{Au}[49,57]$ chemical interactions between the sulphur site of $\alpha-6 \mathrm{~T}$ and gold have been demonstrated by $\mathrm{T}$. Schwieger et al. Also for the interface between $\alpha-6 \mathrm{~T}$ and $\mathrm{Pd}$ a chemical reaction between the $\alpha-6 \mathrm{~T}$ molecules and the substrate during the formation of a monolayer of at-lying $\alpha$ $6 \mathrm{~T}$ is evident from the XPS and UPS measurements [46]. More insight in the importance of the strength of chemical interactions at OSC/metal interfaces can be gained by comparison of the $\alpha-6 \mathrm{~T} / \mathrm{Pd}[46]$ and the $\alpha-6 \mathrm{~T} / \mathrm{Au}[49,57]$ system. UPS measurements reveal for both interfaces an interface dipole of $-1.2 \mathrm{eV}$. In contrast to this similarity, the hole injection barrier for the $\alpha-6 \mathrm{~T} / \mathrm{Au}$ interface is increased by 0.5 $\mathrm{eV}$ with respect to the $\alpha-6 \mathrm{~T} / \mathrm{Pd}$ system. In spite of the fact, that the electrode materials $\mathrm{Pd}$ and Au have a very similar work function they show different electronic properties at the interface to the OSC $\alpha-6 \mathrm{~T}$, which can be explained by the consequence of the strength of the chemical interaction be- tween the OSC and the metal surface at the interface. Relatively small chemical interactions take place at the interface between $\alpha-6 \mathrm{~T}$ and $\mathrm{Au}$. Such a weak-interacting interface can be described within the model of induced density of interface states (IDIS) [50, 58-65]. In the context of the IDIS-model for weak-interacting OSC/metal interfaces, the interface dipole as well as the injection barrier for holes is related to the tendency at $\mathrm{OSC} / \mathrm{metal}$ interfaces to align the charge neutrality level (CNL) of the OSC and the meal work function. This is driven by a finite density of states, which is induced in the gap of the OSC by weak interactions and determines the position of the CNL. The tendency of the CNL to align with the metal work function is screened by the induced density of states. Contrary to the $\alpha-6 \mathrm{~T} / \mathrm{Au}$ system, at the $\alpha-6 \mathrm{~T} / \mathrm{Pd}$ interface the chemical interactions, which can be observed in the way of interface states most likely pin the Fermi level at the interface and thus they predominantly determine the interface energeticss for this system.

The comparison of Fig. (6 and 7) give us a wealth of important and unexpected information about the effect of contact contaminations on the interface characteristics. In all cases a decrease of the interface dipole and the hole injection barrier is observed. This is in agreement with what has been reported for different OSC in contact to $\mathrm{Au}[26,30,31]$. For the different systems the reduction of these two parameters considerably varies. Although for the interfaces with Ag and $\mathrm{Au}$ the variation for the interface dipole and the hole injection barrier is substantial, for the $\alpha-6 \mathrm{~T} / \mathrm{Pt}$ and $\alpha-6 \mathrm{~T} / \mathrm{Pd}$ system mainly a reduction of the interface dipole can be observed accompanied by relatively small decrease in the barrier for hole injection.

The reduction of the interface dipole is predominantly ascribed to the reduction of the metal surface dipole (the socalled Push-Back effect) $[16,19,22]$ due to the presence on the contamination layer on top of the ex-situ cleaned substrate surfaces. This parallels the reduction of the metal work function upon the adsorption of rare gases56. The observed

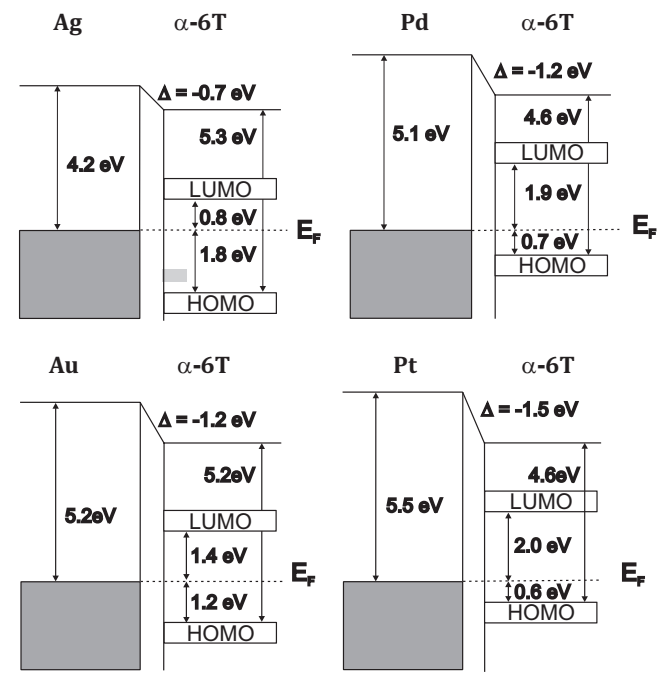

Fig. (6). Interface energeticss for the interfaces of the OSC $\alpha-6 \mathrm{~T}$ in contact to in-situ cleaned, atomically clean metal substrate surfaces ( $\mathrm{Au}, \mathrm{Ag}, \mathrm{Pd}$, and Pt) as obtained by ultraviolet photoemission spectroscopy [Adopted from Ref. 32, 45, 66]. The difference in the ionization energy of about $0.7 \mathrm{eV}$ for the different substrates is due to different molecular orientations of the molecules as discussed in the literature (see Ref. [67-69]). 


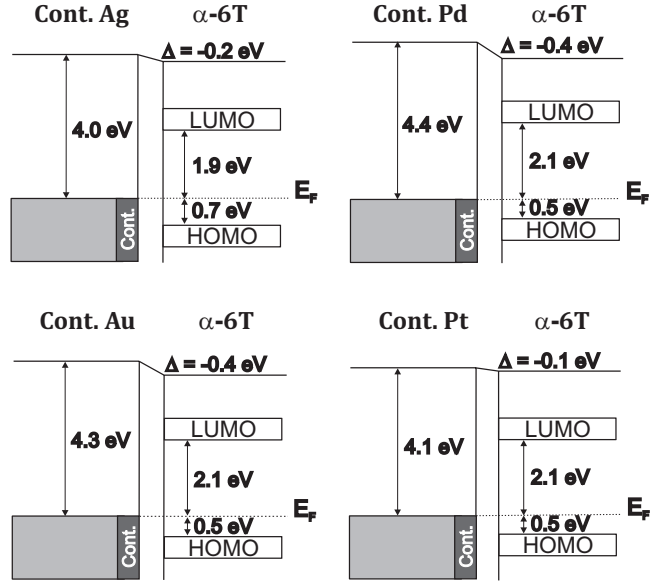

Fig. (7). Schematic energy level diagrams for the interfaces of the OSC $\alpha-6 \mathrm{~T}$ in contact to ex-situ cleaned, contaminated metal substrate surfaces (Au, Ag, Pd, and $\mathrm{Pt}$ ) as obtained by ultraviolet photoemission spectroscopy. With "Cont." the on the surface present contamination layer due to the ex-situ treatment of the electrode surface is denoted (Adopted from Ref. 32, 66).

chemical interactions and the charge transfer at the interfaces of $\alpha-6 \mathrm{~T}$ in contact to $\mathrm{Ag}, \mathrm{Pd}$, and Pt quite likely also contribute to the interface dipole. For contaminated interfaces a finite interface dipole remains in all four cases. This means that the contamination does not electronically decouple the $\alpha-6 T$ molecules and the substrate surface. From Fig. (7) it is evident that for the interfaces using contaminated contacts the hole injection barrier is almost independent from the underlying metal (within an error of $0.2 \mathrm{eV}$ ). Because of the used ex-situ cleaning procedure or the exposure of the substrate to ambient conditions, it is not important which metal one chooses for the injection of hole due to very similar interface electronic properties. Consequently, the position of the Fermi level becomes independent of the underlying metal substrate, that is, the metals become indistinguishable or faceless.

Thus, contact contaminations of the electrodes essentially affect the interface energeticss in particular the electrode work function, the interface dipole, and the hole injection barrier [28-30, 32, 66] and therefore the performance of organic electronic devices $[29,30]$. These new insights in the interface energetics at so-called realistic OSC/metal interfaces can help in the future to better design and optimize organic electronic devices.

\section{INTERFACES RELEVANT FOR ORGANIC SPIN- TRONIC DEVICES}

In the last years different pioneering experimental works $[47,70]$ suggested that OSCs can also be useful as a major opportunity for application in the growing field of organic spin electronics [48], also referred to as organic spintronic. Organic spintronics represents the synopsis of OSC material for the transport and the control of spinpolarized information. OSC mainly consists of light elements leading to a weak spin-orbit coupling. Despite the presence of nuclear spins, the hyperfine interaction in organic materials is also weak48. Therefore, OSC are believed to have a large spin diffusion length $\lambda_{S}$ and a long spin relaxation time [71, 72] showing the great potential of OSC for their use in organic spintronic devices. For the injection and the detection of spin-polarized electrons in an OSC, a ferromagnetic (FM) electrode with a substantial degree of electron spinpolarization of the electrons in the conduction band is required. Possible FM electrode materials, which are used in organic spintronic devices, are lanthanum strontium manganite oxides ( $\left.\mathrm{La}_{0.7} \mathrm{Sr}_{0.3} \mathrm{MnO}_{3}, \mathrm{LSMO}\right)$ and Co. LSMO represent an attractive rare-earth compound which is characterized by a nearly $100 \%$ spin-polarization of the charge carriers at room temperature [73]. Consequently, in many organic spintronic devices LSMO films are used as injector for spinpolarized electrons $[47,70,74,75]$, and the electronic structure of $\mathrm{La} 0: 7 \mathrm{Sr} 0: 3 \mathrm{MnO} 3$ thin films for spintronic application has been studied in detail [76]. Also Co is a commonly used electrode material for the injection and detection of spinpolarized charge carriers in organic spintronic devices [70, 77-79] due to its high bulk spin-polarization of about $45 \%$ $[48,80,81]$. A lot of activity is presently going on to inject spin-polarized currents in OSC [47, 70, 75, 77-80, 82-84].

By today only a few studies on interfacial properties of spintronic relevant contact materials (e.g. $\mathrm{La}_{0.7} \mathrm{Sr}_{0.3} \mathrm{MnO}_{3}$, $\mathrm{Co}$, and $\mathrm{Fe}$ ) and different organic semiconductors have been published using PES. In this context of organic spintronic interfaces several OSC/electrode systems have been studied under different points of view: (i) interfaces between different OSCs and LSMO [85-88], Co [80, 86, 88-91], and $\mathrm{Fe}$ [91,92] to obtain information about the charge transport across such interfaces, (ii) the effect of surface contaminations that often exists during device fabrication on the energy level alignment of organic spintronic relevant interfaces [33, $34,66,87]$, and (iii) the role of a thin tunnel barrier of $\mathrm{Al}_{2} \mathrm{O}_{3}$ between the OSCs and a Co contact $[34,88,89]$ to overcome the conductivity mismatch problem.

To address the effect of a contamination layer at the electrode surface for organic spintronic devices we used combined X-ray and ultraviolet photoemission spectroscopy to investigate the resulting interface energeticss for $\mathrm{CuPc} /$ LSMO and $\alpha-6$ T/LSMO interfaces prior and after the exposure to ambient conditions $[33,87,93]$. Clean LSMO contact surfaces have been prepared by annealing in an oxygen atmosphere after a pre-treatment with acetone. The contaminated LSMO substrates have been cleaned with an acetone treatment after the LSMO films have been exposed to ambient conditions representing a cleaning procedure which is also applied in the fabrication of organic spintronic devices using LSMO as bottom electrodes [47, 70, 74, 75, 83]. For further details of the applied in-situ and ex-situ cleaning procedures we refer the reader to previous publications [33, 87]. From the top panels, the presence of a surface contamination layer on top of the LSMO films after the ex-situ treatment is clearly evident from the behaviour of the $\mathrm{C} 1 s$ and the $\mathrm{O} 1 s$ excitation feature. In Fig. (8) the $\mathrm{C} 1 s$ and $\mathrm{O} 1 s$ core level spectra measured from a LSMO surface for an ex-situ cleaning procedure (top panels) and after applying the in-situ annealing treatment (bottom panels) are depicted. From the core level analysis a contamination layer consisting of carbon $(74 \%)$ and oxygen $(26 \%)$ is identified for the ex-situ cleaned samples. Upon applying the in-situ annealing procedure in an oxygen atmosphere, the features in the $\mathrm{C} 1 s$ and $\mathrm{O} 1 s$ core level excitations, respectively, resulting from the contamination layer at the LSMO surface have vanished. 


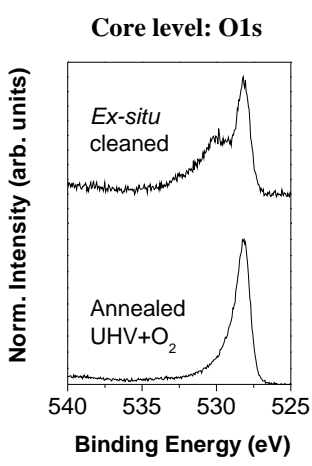

(a)

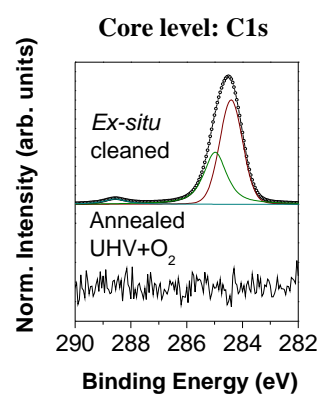

(b)
Fig. (8). O1s (panel (a)) and the $\mathrm{C} 1 s$ (panel (b)) core level excitation from an ex-situ (top panels) and an in-situ (bottom panels) treated LSMO surface (Adopted from Ref. 93).

This indicates that the contaminations due to carbon, carbonates as well as adsorbed water species are indeed removed.

In Fig. (9) the positions of the molecular levels at $\mathrm{CuPc}$ and $\alpha-6 \mathrm{~T}$ interfaces with atomically clean and contaminated LSMO surfaces are shown. The work function of the air exposed and ex-situ cleaned, contaminated LSMO surface is significantly reduced $(4.0-4.2 \mathrm{eV})$ with respect to the that of the in-situ treated, clean surface (4.8-4.9 eV). The reduction of the work function of this potential electrode material for organic spintronic devices due to the presence of the contamination layer at ex-situ cleaned, contaminated surfaces emphasizes the vital importance of the preparation conditions on the interface energeticss in organic spintronic devices. In terms of interface energeticss, the most striking cleaning-procedure induced observation is the systematic variation of the energy level alignment as a function of this surfaces contamination. When the contact surface is contaminated both interfaces to the OSCs CuPc and $\alpha-6 \mathrm{~T}$ show significantly enhanced barriers for the hole injection across the corresponding OSC/contaminated LSMO contact, whereas the barrier for electron injection is lowered with respect to the OSC/clean LSMO interfaces. In a recent publication F. Li et al. [94] applied different ex-situ cleaning procedures to $\mathrm{La}_{0.7} \mathrm{Sr}_{0.3} \mathrm{MnO}_{3}$ surfaces to remove the surface contaminations and to increase the oxygen stoichiometry at the LSMO surface, i.e. ultrasonic treatment in organic solvents, LSMO heated in the so-called TL1 solution [95]. The work function studied with ultraviolet photoemission spectroscopy for LSMO substrates treated by the TL1 solution varies between $4.6-4.9 \mathrm{eV}$ depending on the treatment time. The LSMO work function and as consequence the interface energeticss at such interfaces considerably depends on the LSMO pre-treatment, i.e. whether there is still a contamination layer present at the LSMO surface.

The fabrication of a thin tunnel barrier is often important to overcome the so-called conductivity mismatch $[48,96]$ between the FM electrode and the OSC spacer, which is a problem in OSC spintronic devices $[96,97]$. The insertion of a thin tunnel barrier of $\mathrm{Al}_{2} \mathrm{O}_{3}$ as a large resistance in between the FM electrode and the OSC has been successfully applied in organic spintronic devices [78, 79]. The influence of such a thin $\mathrm{Al}_{2} \mathrm{O}_{3}$ buffer layer on the interface electronic properties of interfaces between the OSC $\mathrm{Alq}_{3}$ and a Co electrode

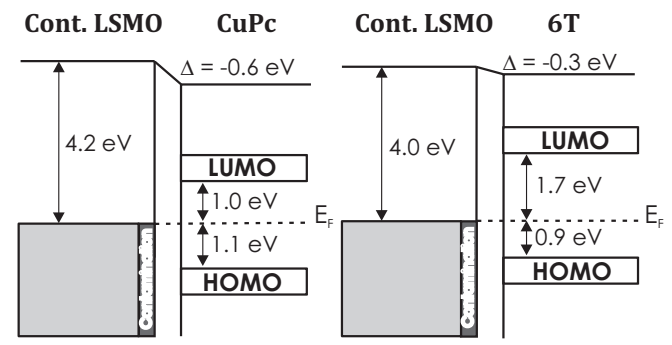

(a)

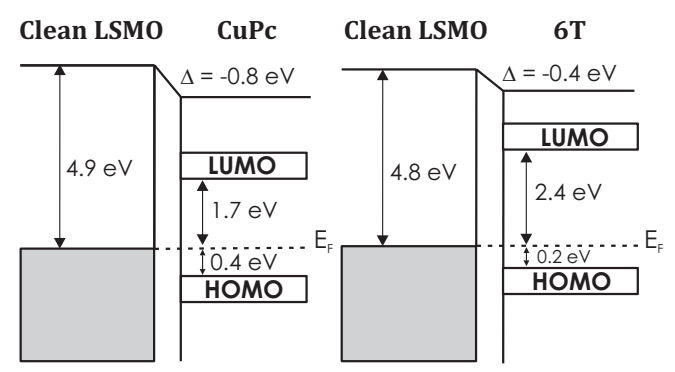

(b)

Fig. (9). Schematic energy level diagrams of the OSC CuPc and $\alpha$ $6 \mathrm{~T}$ in contact to ex-situ (panel a) and in-situ pre-treated (panel b) $\mathrm{La} 0.7 \mathrm{Sr} 0.3 \mathrm{MnO} 3$ contacts [(Adopted from Ref. 33, 40, 87)].

has been studied by means of photoemission spectroscopy by Y. Q. Zhan et al. [86, 98].

Y. Q. Zhan and co-workers investigated in 2008 [86] the interfacial properties at $\mathrm{Alq}_{3} / \mathrm{Co}$ interfaces. They observed an interfacial dipole of $-1.5 \mathrm{eV}$. By using the optical gap of 2.8 $\mathrm{eV}$ [99] or the HOMO-LUMO distance directly measured by STS (scanning tunneling spectroscopy) of $2.96 \mathrm{eV}$ [100] to estimate the HOMO-LUMO gap, the barrier for electron injection is much smaller than for the injection of holes. For this reason the dominant charge carriers in $\mathrm{Co} / \mathrm{Alq}_{3} / \mathrm{LSMO}$ spin valve devices should be electrons. Additionally performed XPS measurements demonstrate the penetration of $\mathrm{Alq}_{3}$ films with $\mathrm{Co}$ atoms when Co is deposited on top of the OCS. A chemical reaction between the Co atoms and the phenoxide part of $\mathrm{Alq}_{3}$ molecules and near the interface have been observed. And in 2009 Y. Q. Zhan and co-workers [98] investigated the electronic structure of the $\mathrm{Co} / \mathrm{Al}_{2} \mathrm{O}_{3} / \mathrm{Alq}_{3}$ interface using photoemission spectroscopy (XPS,UPS) and observed a strong interface dipole of $-1.3 \mathrm{eV}$, which leads to a reduction of the injection barrier for electrons and an increase in the hole injection barrier. The core level excitations $\mathrm{O} 1 s$ and $\mathrm{Co} 2 p$ indicate that the insertion of the thin tunnel barrier of $\mathrm{Al}_{2} \mathrm{O}_{3}$ separates the $\mathrm{Alq}_{3}$ thin film and Co electrode and prevent a chemical reaction between them. The interface energetics of the $\mathrm{Co} / \mathrm{Al}_{2} \mathrm{O}_{3} / \mathrm{Alq}_{3}$ was studied using UPS and a work function for the bare, as-deposited Co films was estimated to be $5 \mathrm{eV}$. The work function of the inverted structure, $\mathrm{Alq}_{3} / \mathrm{Al}_{2} \mathrm{O}_{3} / \mathrm{Co}$ [106-108] is $3.7 \mathrm{eV}$ resulting in an interfacial dipole of $-1.3 \mathrm{eV}$ for the $\mathrm{Co} / \mathrm{Al}_{2} \mathrm{O}_{3} / \mathrm{Alq}_{3}$ interface, which leads to an increase in the hole injection barrier and decrease in the barrier for electron injection. This interface dipole is similar to that which has been observed for the $\mathrm{Co} / \mathrm{Alq}_{3}$ interface [86]. Additionally performed XMCD (Xray magnetic dichroism) measurements demonstrate that a thin tunnel barrier of $\mathrm{Al}_{2} \mathrm{O}_{3}$ between of the OSC and the $\mathrm{Co}$ electrode leads to better magnetic ordering at the interface. This suggests a better performance of organic spintronic devices with such a thin tunnel barrier used to overcome the 
conductivity mismatch. Y. Q. Zhan and co-workers $[86,98]$ could show that the observed interface energeticss with an inserted $\mathrm{Al}_{2} \mathrm{O}_{3}$ bu_er layer is consistent with the results from V. Dediu et al. [78] and T.S. Santos et al. [79]. For the OSC rubrene Shim et al. [72] reported in 2008 a large spin diffusion length of $13.3 \mathrm{~nm}$ in amorphous rubrene, showing the great potential of OSC for organic spintronic devices. The OSC rubrene and pentacene are also characterized by large charge carrier mobilities, for organic standards. For rubrene high hole mobilities in the range of $15-20 \mathrm{~cm} 2 / \mathrm{Vs}$ were reported for high-purity single crystals [101]. Also pentacene exhibits high charge carrier mobilities from typical 0.4-1 $\mathrm{cm}^{2} / \mathrm{Vs}$ for thin film transistors (TFTs) made from good quality pentacene films [102] to $35 \mathrm{~cm}^{2} / \mathrm{Vs}$ for high-purity single-crystals [103]. Consequently, rubrene and pentacene are promising candidates for future electronic devices as well as for the investigation of spin transport properties. The choice of $\mathrm{Co}$ as electrode material comes from the fact that Co is a commonly used ferromagnetic source for the injection as well as detection of spin-polarized electrons in spintronic devices $[70,78,79]$ with a bulk spin-polarization of around $45 \%$ [48, 80, 81]. A common problem for spin injection and detection using a ferromagnetic metal (such as Co) electrode is the so-called conductivity mismatch $[48,96,97]$. As already mentioned the fabrication of a tunnel barrier, $\mathrm{Al}_{2} \mathrm{O}_{3}$, is important to overcome the conductivity mismatch between the metal electrodes and a semiconductor spacer, which is a problem in semiconductor spintronic devices $[96,97]$.

We studied the interface electronic properties of ex-situ, acetone cleaned $\mathrm{Co}$ and $\mathrm{Al}_{2} \mathrm{O}_{3} / \mathrm{Co}$ contacts to the OSC pentacene and rubrene by combined X-ray and ultraviolet photoemission spectroscopy [34] to investigate the influence of the applied ex-situ cleaning procedure on the energy level alignment. By using the ex-situ, acetone cleaning procedure a contamination layer, which consists of carbon and oxygen still remains on top of the $\mathrm{Co}$ and $\mathrm{Al}_{2} \mathrm{O}_{3} / \mathrm{Co}$ films. The Co2p, $\mathrm{C} 1 s$, and $\mathrm{O} 1 s$ core level excitations of the ex-situ cleaned Co contacts indicate that the contaminations on top of ex-situ cleaned Co surfaces results in a native cobalt oxide layer. From the performed core level measurements (Co2p, A12p) in dependence of the thickness of the OSC overlayer it is evident that the contamination layer is closed and prevents chemical reactions between the substrates and pentacene and rubrene, respectively. In Fig. (10) the schematic energy level diagrams of contaminated interfaces between $\mathrm{Co}$ and $\mathrm{Al}_{2} \mathrm{O}_{3} / \mathrm{Co}$ electrodes and the OSC pentacene and rubrene are summarized. For ex-situ cleaned Co contacts a substantially reduced work function of $4.3 \mathrm{eV}$ was determined with respect to the work function of atomically clean, polycrystalline Co surfaces $(5.0-5.1 \mathrm{eV})$ [86, 89, 90, 98, 104]. In the case of ex-situ cleaned $\mathrm{Al}_{2} \mathrm{O}_{3} / \mathrm{Co}$ surfaces a work function ranging from $3.1 \mathrm{eV}$ to $3.5 \mathrm{eV}$ was measured. In contrast to the interfaces of the OSC pentacene to clean $\mathrm{Al}_{2} \mathrm{O}_{3} / \mathrm{Co}$ and clean Co contacts $[89,105]$ an increase of the hole injection barrier for the $3 \mathrm{~nm}$ thick $\mathrm{Al}_{2} \mathrm{O}_{3}$ tunnel barrier was observed. For all investigated interfaces a very small, short range interface dipole was observed suggesting that the interface dipole is hardly influenced by the introduction of the thin tunnel barrier of $\mathrm{Al}_{2} \mathrm{O}_{3}$. Furthermore it is expected for OSC spintronic devices from the very high hole injection barrier that only little charge carriers will be able to tunnel trough the interface.

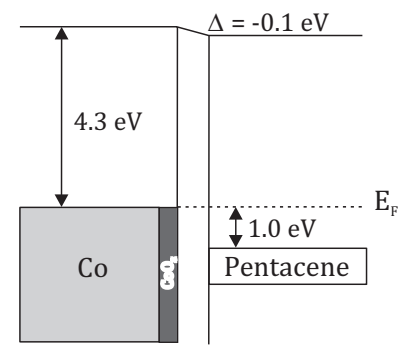

(a)

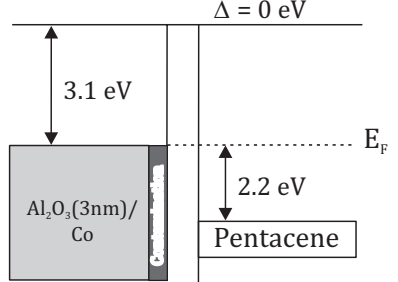

(b)

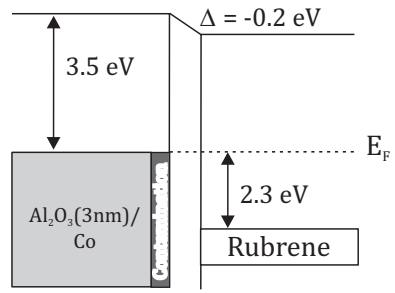

(c)

Fig. (10). Comparison of the electronic structure of vacuum deposited pentacene thin films on top ex-situ cleaned Co and A12O3 electrode surfaces (panels $\mathbf{a}$ and $\mathbf{b}$ ). Panel $\mathbf{c}$ depicts the energy level alignment for the contaminated rubrene $/ \mathrm{Al}_{2} \mathrm{O}_{3} / \mathrm{Co}$ interface, as determined by UPS (Adopted from Ref. 34).

\section{SUMMARY}

During the last decades there has been considerable progress as regards the understanding of OSC/electrode interfaces. In this review the influence of remaining contamination on the substrate surface due to the applied cleaning procedure on the energy level alignment of OSC/electrode interfaces has been addressed. Contact contaminations of the electrodes essentially affect the interface energeticss in particular the interface parameters electrode work function, interface dipole, and hole injection barrier and therefore the performance of organic electronic devices. These new insights in the interface energeticss at so-called realistic $\mathrm{OSC} /$ metal interfaces can help in the future to better design and optimize organic electronic devices such as OLEDs, OFETS, and OPVs. The reviewed investigations of the interface energeticss of interfaces relevant for organic spintronic devices, and in particular the effect of contamination layer induced changes in the energy level alignment of such interfaces lead to a significant progress in the understanding of the interfacial properties of organic spintronic relevant interfaces.

They represent one step toward the description of spin transport in organic spintronic devices. This represents essential information of the use of OSCs in organic spintronic devices.

\section{ACKNOWLEDGEMENTS}

We thank R. B. Gangineni, K. Dörr, W. J. M. Naber, W. G. van der Wiel, and C. Schmidt for fruitful discussions and their contribution to some of the experiments. Technical support of R. Hübel, S. Leger, R. Schönfelder, and J. G. Sanderinck is gratefully acknowledged. Part of the work has been financially supported by the Deutsche Forschungsgemeinschaft (project No. KN393/5, KN393/9, and KN393/14). 


\section{REFERENCES}

[1] Brütting W. Physics of Organic Semiconductors. WILEY-VCH Verlag: Weinheim 2005.

[2] Brütting W, Rieß W. Grundlagen der organischen Halbleiter. Phys J 2008; 7: 33 .

[3] Klauk H. Organic Electronics. WILEY-VCH Verlag: Weinheim 2007.

[4] Tang CW, VanSlyke SA. Organic electroluminescent diodes. Appl Phys Lett 1987; 51: 913.

[5] Blochwitz J, Fritz T, Pfeiffer M, et al. Interface electronic structure of organic semiconductors with controlled doping levels. Org Electr 2001; 2: 97.

[6] Walzer K, Maennig B, Pfeiffer M, Leo K. Highly efficient organic devices based on electrically doped transport layers. Chem Rev 2007; 107: 1233.

[7] Koch N. Organic electronic devices and their functional interfaces. ChemPhysChem 2007; 8: 1438.

[8] Müllen K, Scherf U. Organic light emitting devices. WILEY-VCH Verlag: Weinheim 2006.

[9] Horowitz G. Organic thin film transistors: from theory to real devices. J Mater Res 2004; 19: 1946.

[10] Clemens W, Fix I, Ficker J, Knobloch A, Ullmannn A. From polymer transistors toward printed electronics. J Mater Res 2004; 19: 1963.

[11] Scheinert S, Paasch G. Fabrication and analysis of polymer fieldeffect transistors. Phys Status Solidi A 2004; 6: 1263.

[12] Cicoira F, Santato C. Organic light emitting field effect transistors: advances and perspectives. Adv Funct Mater 2007; 17: 3421.

[13] Rand BP, Genoe J, Heremans P, Poortmans J. Solar cells utilizing small molecular weight organic semiconductors. Prog Photovolt 2007; 15: 659.

[14] Maennig B, Drechsel J, Gebeyehu D, et al. Organic p-i-n solar cells. Appl Phys A 2004; 79: 1.

[15] Peumans P, Bulovic V, Forrest SR. Efficient photon harvesting at high optical intensities in ultrathin organic double-heterostructure photovoltaic diodes. Appl Phys Lett 2000; 76: 2650.

[16] Ishii H, Sugiyama K, Ito E, Seki K. Energy level alignment and interfacial electronic structure at organic/metal and organic/organic interfaces. Adv Mater 1999; 11: 605.

[17] Salaneck WR, Seki K, Kahn A, Pireaux JJ. Conjugated polymer and molecular interfaces: Science and Technology for photonic and optoelectronic applications. New York: Marcel Dekker 2002 [and references therein].

[18] Kahn A, Koch N, Gao W. Electronic structure and electrical properties of interfaces between metals and $\pi$-conjugated molecular films. J Polym Sci B 2003; 41: 2529.

[19] Crispin X. Interface dipole at organic/metal interfaces and organic solar cells. Solar Energy Mater Solar Cells 2004; 83: 147.

[20] Scott JC. Metal-organic interface and charge injection in organic electronic devices. J Vac Sci Technol A 2003; 21: 521.

[21] Knupfer M, Peisert H. Electronic properties of interfaces between model organic semiconductors and metals. Phys Status Solidi A 2004; 201: 1055.

[22] Knupfer M, Paasch G. Origin of the interface dipole at interfaces between undoped organic semiconductors and metals. J Vac Sci Technol A 2005; 23: 1072.

[23] Fahlman M, Crispin A, Crispin X, et al. Electronic structure of hybrid interfaces for polymer-based electronics. J Phys Condens Matter 2007; 19: 183202.

[24] Koch N. Energy levels at interfaces between metals and conjugated organic molecules. J Phys Cond Matter 2008; 20: 184008.

[25] Braun S, Salaneck WR, Fahlman M. Energy-Level alignment at organic/metal and organic/organic interfaces. Adv Mater 2009; 21: 1450 .

[26] Hwang J, Wan A, Kahn A. Energetics of metal-organic interfaces: New experiments and assessments of the field. Mater Sci Eng R 2009; 64: 1 .

[27] Gao Y. Surface analytical studies of interfaces on organic semiconductor devices. Mater Sci Eng R 2010; 68: 39.

[28] Narioka S, Ishii H, Yoshimura D, et al. The electronic structure and energy level alignment of porphyrin/metal interfaces studied by ultraviolet photoelectron spectroscopy. Appl Phys Lett 1995; 67: 1899.
Shen C, Hill IG, Kahn A. Role of electrode contamination in electron injection at $\mathrm{Mg}: \mathrm{Ag} / \mathrm{Alq}_{3}$ interfaces. Adv Mater 1999; 11: 1523.

[30] Wan A, Hwang J, Amy F, Kahn A. Impact of electrode contamination on the alpha-NPD/Au hole injection barrier. Org Electr 2005; 6: 47.

[31] Wan A. Ph.D. thesis, Princeton University 2006.

[32] Grobosch M, Knupfer M. Charge-Injection barriers at metals become faceless. Adv Mater 2007; 19: 754.

[33] Grobosch M, Dörr K, Gangineni RB, Knupfer M. Energy level alignment and injection barriers at spin injection contacts between $\mathrm{La}_{0.7} \mathrm{Sr}_{0.3} \mathrm{MnO}_{3}$ and organic semiconductors. Appl Phys Lett 2008; 92: 023302 .

[34] Grobosch M, Schmidt C, Naber WJM, van der Weil WG, Knupfer M. A photoemission study of interfaces between organic semiconductors and $\mathrm{Co}$ as well as $\mathrm{Al}_{2} \mathrm{O}_{3} / \mathrm{Co}$ contacts. Synth Met 2010; 160: 238.

[35] Lüth H. Surfaces and interfaces of solids. In surface science springer series, vol. 15. Berlin: Springer-Verlag 1993.

[36] Hüfner S. Photoelectron spectroscopy: principles and applications. Berlin: Springer 1995.

[37] Briggs D, Grant JT. Surface analysis by Auger and X-Ray photoelectron spectroscopy. IM Publications 2003.

[38] Cahen D, Kahn A. Electron energetic at surfaces and interfaces: concepts and experiments. Adv Mater 2003; 15: 271.

[39] Alfredsson Y. Ph.D. thesis 2005, Uppsala Universitet.

[40] Grobosch M. Ph.D. thesis 2009, Technical University Dresden.

[41] Seah MP, Dench WA. Quantitative electron spectroscopy of surfaces: a standard data base for electron inelastic mean free paths in solids. Surf Interface Anal 1979; 1: 2.

[42] Hill IG, Kahn A, Soos ZG, Pascal Jr. RA. Charge-separation energy in films of $\pi$-conjugated organic molecules. Chem Phys Lett 2000; 327: 181.

[43] Schwieger T, Peisert H, Golden MS, Knupfer M, Fink J. Electronic structure of the organic semiconductor copper phthalocyanine and $\mathrm{K}-\mathrm{CuPc}$ studied using photoemission spectroscopy. Phys Rev B 2000; 66: 155207.

[44] Knupfer M, Pichler T, Golden MS, et al. Size of electron-hole pairs in $\pi$-conjugated systems. Phys Rev Lett 1999; 83: 1443.

[45] Grobosch M, Knupfer M. Energy level alignment and interface states at $\alpha$-sexithiophene/Ag interfaces. Org Electr 2007; 8: 235.

[46] Grobosch M, Knupfer M. Electronic properties of the interface between the organic semiconductor $\alpha$-sexithiophene and polycrystalline palladium. Org Electr 2008; 9: 767.

[47] Dediu V, Murgia M, Matacotta FC, Taliani C, Barbanera S. Room temperature spin polarized injection in organic semiconductor Solid State Commun 2002; 122: 181.

[48] Naber WJM, Faez S, av der Wiel WG. Organic spintronics. J Phys D: Appl Phys 2007; 40: R205-R228.

[49] Schwieger T, Liu X, Peisert H, Adolphi B, Kiriy N, Knupfer M. Electronic properties of interfaces between different sexithiophenes and gold. J Appl Phys 2005; 97: 123712.

[50] Vazquez H, Gao W, Flores F, Kahn A. Energy level alignment at organic heterojunctions: role of the charge neutrality level. Phys Rev B 2005; 71: 041306.

[51] Salaneck WR, Fahlman M. Hybrid interfaces of conjugate polymers: band edge alignment studied by ultraviolet photoelectron spectroscopy. J Mater Res 2004; 19: 1917.

[52] Koch N, Kahn A, Ghijsen J, et al. Conjugated organic molecules on metal versus polymer electrodes: demonstration of a key energy alignment mechanism. Appl Phys Lett 2003; 82: 70.

[53] Crispin X, Geskin V, Crispin A, et al. Characterization of the interface dipole at organic/metal interfaces. J Am Chem Soc 2002; 124: 8131.

[54] Peisert H, Knupfer M, Fink J. Energy level alignment at organic/metal interfaces: dipole and ionization potential. Appl Phys Lett 2002; 81: 2400.

[55] Yan L, Watkins NJ, Zorba S, Gao YL, Tang CW. Thermodynamic equilibrium and metal-organic interface dipole. Appl Phys Lett 2002; 81: 2752.

[56] Wandelt K. The local work function: concept and implications. Appl Surf Sci 1997; 111: 1.

[57] Knupfer M, Liu X. Interface electronic properties of oligothiophenes: the effect of chain length and chemical substituent. Surf Sci 2006; 600: 3978 . 
[58] Vazquez H, Oszwaldowski R, Pou P, et al. Dipole formation at metal/PTCDA interfaces: role of the charge neutrality level. Europhys Lett 2004; 65: 802 .

[59] Vazquez H, Flores F, Oszwaldowski R, Ortega J, Perez R, Kahn A. Barrier formation at metal-organic interfaces: dipole formation and the charge neutrality level. Appl Surf Sci 2004; 234: 107.

[60] Vazquez H, Flores F, Kahn A. Induced density of states model for weakly-interacting organic semiconductor interfaces. Org Electr 2007; 8: 241.

[61] Molodtsova OV, Grobosch M, Knupfer M, Aristov VY. Consistent experimental determination of the charge neutrality level and the pillow effect at metal/organic interfaces. Appl Phys Lett 2007; 91: 244103.

[62] Vazquez H, Dappe YJ, Ortega J, Flores F. Energy level alignment at metal/organic semiconductor interfaces: "Pillow" effect, induced density of interface states, and charge neutrality level. J Chem Phys 2007; 126: 144703.

[63] Vazquez H, Dappe YJ, Ortega J, Flores F. A unified model for metal/organic interfaces: IDIS, "pillow" effect and molecular permanent dipoles. Appl Surf Sci 2007; 254: 378.

[64] Flores F, Ortega J, Vazquez H. Modelling energy level alignment at organic interfaces and density functional theory. Phys Chem Chem Phys 2009; 11: 8658.

[65] Abad E, Ortega J, Flores F. Density functional theory calculations and the induced density of interface states model for noble metals/C 60 interfaces. J Vac Sci Technil B 2009; 27: 2008.

[66] Grobosch M, Dörr K, Gangineni RB, Knupfer M. Alignment of the energy levels and charge injection barriers at interfaces for spin injection: $\mathrm{La}_{0.7} \mathrm{Sr}_{0.3} \mathrm{MnO}_{3}$ in contact to organic semiconductors. Phys Stat Solidi B 2008; 245: 799.

[67] Ivanco J, Haber T, Resel R, Netzer FP, Ramsey MG. Electronic and geometric structure of electro-optically active organic films and associated interfaces. Thin Solid Films 2006; 514: 156.

[68] Ivanco J, Netzer F, Ramsey MG. Dissociation of sexithiophene on Al(111) surface. Org Electr 2007; 8: 545.

[69] Ivanco J, Haber T, Krenn JR, Netzer FP, Resel R, Ramsey MG. Sexithiophene films on ordered and disordered $\mathrm{TiO}_{2}(110)$ surfaces: electronic, structural and morphological properties. Surf Sci 2007; 601: 178.

[70] Xiong ZH, Wu D, Vardeny ZV, Shi J. Giant magnetoresistance in organic spin-valves. Nature 2004; 427: 821.

[71] Drew AJ, Hoppler J, Schulz L, et al. Direct measurement of the electron spin diffusion length in a fully functional organic spin valve by low-energy muon spin rotation Nat Mater 2009; 8: 109.

[72] Shim JH, Raman KV, Park YJ, et al. Large spin diffusion length in an amorphous organic semiconductor. Phys Rev Lett 2008; 100: 226603.

[73] Park JH, Vescovo E, Kim HJ, Kwon C, Ramesh R, Venkatesan T. Direct evidence for a half-metallic ferromagnet. Nature 1998; 392: 794.

[74] Majumdar S, Laiho R, Laukkanen P, Vayrynen IJ, Majumdar HS, Osterbacka R, Application of regioregular polythiophene in spintronic devices: effect of interface. Appl Phys Lett 2006; 89: 122114.

[75] Pang ZY, Chen YX, Liu TT, et al. Giant magnetoresistance in $\mathrm{La}_{0.67} \mathrm{Ca}_{0.33} \mathrm{MnO}_{3} / \mathrm{Alq}_{3} / \mathrm{Co}$ sandwiched-structure organic spin values. Chin Phys Lett 2006; 23: 1566.

[76] de Jong MP, Dediu VA, Taliani C, Salaneck WR. Electronic structure of $\mathrm{La}_{0.7} \mathrm{Sr}_{0.3} \mathrm{MnO}_{3}$ thin films for hybrid organic/inorganic spintronics applications. J Appl Phys 2003; 94: 7292.

[77] Naber WJM, Craciun MF, Lemmens JHJ, et al. Controlled tunnelcoupled ferromagnetic electrodes for spin injection in organic single-crystal transistors. Org Electr 2010; 11: 743.

[78] Dediu V, Hueso LE, Bergenti I, et al. Room-temperature spintronic effects in $\mathrm{Alq}_{3}$-based hybrid devices. Phys Rev B 2008; 78 : 115203.

[79] Santos TS, Lee JS, Migdal P, Lekshmi IC, Satpati B, Moodera JS. Room-Temperature Tunnel Magnetoresistance and Spin-Polarized Tunneling through an Organic Semiconductor Barrier. Phys Rev Lett 2007; 98: 016601.

[80] Cinchetti M, Heimer K, Wüstenberg JP, et al. Determination of spin injection and transport in a ferromagnet/organic semiconductor heterojunction by two-photon photoemission. Nat Mater 2009; 8: 115 .
[81] Andreyev O, Koroteev YM, Albaneda MS, et al. Spin-resolved two-photon photoemission study of the surface resonance state on Co/Cu (001). Phys Rev B 2006; 74: 19516.

[82] Shikoh E, Fujiwara A, Ando Y, Miyazaki T. Spin injection into organic light-emitting devices with ferromagnetic cathode and effects on their luminescence properties. Jpn J Appl Phys 2006; 45: 6897.

[83] Majumdar S, Majumdar HS, Laiho R, Osterbacka R. Comparing small molecules and polymer for future organic spin-valves. J Alloys Compd 2006; 423: 169.

[84] Xu W, Szulczewski GJ, LeClair P, et al. Tunneling magnetoresistance observed in $\mathrm{La}_{0.67} \mathrm{Sr}_{0.33} \mathrm{MnO}_{3} /$ organic molecule/Co junctions. Appl Phys Lett 2007; 90: 072506.

[85] Zhan YQ, Bergenti I, Hueso LE, Dediu V, de Jong MP, Li ZS. Alignment of energy levels at the $\mathrm{Alq}_{3} / \mathrm{La}_{0.7} \mathrm{Sr}_{0.3} \mathrm{MnO}_{3}$ interface for organic spintronic devices. Phys Rev B 2007; 76: 045406.

[86] Zhan YQ, de Jong MP, Li FH, Dediu V, Fahlman M, Salaneck WR. Phys Rev B 2008; 78: 045208.

[87] Grobosch M, Dörr K, Gangineni RB, Knupfer M. Energy level alignment and interactions at potential contacts for spin injection into organic semiconductors. Adv Eng Mater 2009; 11: 285.

[88] Borgatti F, Bergenti I, Bona F, et al. Understanding the role of tunnelling barriers in organic spin valves by hard X-ray photoelectron spectroscopy. Appl Phys Lett 2010; 96: 043306.

[89] Popinciuc M, Jonkman HT, van Wees BJ. Energy level alignment symmetry at Co/pentacene/Co interfaces. J Appl Phys 2006; 100: 093714.

[90] Tiba MV, de Jonge WJM, Koopmans B, Jonkman HT. Morphology and electronic properties of the pentacene on cobalt interface. J Appl Phys 2006; 100: 093707.

[91] Aristov VY, Molodtsova OV, Ossipyan VA, Doyle BP, Nannarone S, Knupfer M. Ferromagnetic cobalt and iron top contacts on an organic semiconductor: evidence for a reacted interface. Org Electr 2009; 10: 8 .

[92] Zhan Y, Holmström E, Lizarraga R, et al. Efficient spin injection trough exchange coupling at organic semiconductor/ferromagnetic heterojunctions. Adv Mater 2009; 21: 1 .

[93] Grobosch M, Dörr K, Gangineni RB, Knupfer M. Energy level alignment at interfaces between organic semiconductors and clean ferromagnetic $\mathrm{La}_{0.7} \mathrm{Sr}_{0.3} \mathrm{MnO}_{3}$ thin film contacts for spin injection. Appl Phys A 2009; 95: 95.

[94] Li F, Graziosi P, Tang Q, et al. Electronic structure and molecular orientation of pentacene thin films on ferromagnetic $\mathrm{La}_{0.7} \mathrm{Sr}_{0.3} \mathrm{MnO}_{3}$. Phys Rev B 2010; 81: 205145.

[95] Andersson A, Johannson N, Bröms P, Yu N, Lupo D, Salaneck WR. Fluorine tin oxide as an alternative to indium tin oxide in polymer LEDs. Adv Mater 1998; 10: 859.

[96] Fert A, Jaffrés. Conditions for efficient spin injection from a ferromagnetic metal into a semiconductor. Phys Rev B 2001; 64: 184420 .

[97] Schmidt G, Ferrand D, Molenkamp LW, Filip AT, van Wees BJ. Fundamental obstacle for electrical spin injection from a ferromagnetic metal into a diffusive semiconductor. Phys Rev B 2000; 62: R4790.

[98] Zhan YQ, Kiu XY, Carlegrim E, et al. The role of aluminium oxide buffer layer in organic spin-valve performance. Appl Phys Lett 2009; 94: 053301.

[99] Lee ST, Hou XY, Mason MG, Tang CW. Energy level alignment at Alq/metal interfaces. Appl Phys Lett 1998; 72; 1593.

[100] Alvarado SF, Libioulle L, Seidler PF. STM-excited luminescence on organic materials. Synth Met 1997; 91: 69.

[101] Podzorov V, Menard E, Borissov A, Kiryukhin V, Rogers JA, Gershenson ME. Intrinsic charge transport on the surface of organic semiconductors. Phys Rev Lett 2004; 93: 086602.

[102] Knipp D, Street RA, Krusor B, Apte R, Ho J. Polycrystalline pentacene thin films for large area electronic applications. J Non-Cryst Solids 2002; 299-302:1042.

[103] Jurchescu OD, Baas J, Palstra TTM. Effect of impurities on the mobility of single crystal pentacene. Appl Phys Lett 2004; 84: 3061.

[104] Michaelson HB. Work function of the elements and its periodicity. J Appl Phys 1977; 48: 4729.

[105] Popinciuc M, Jonkman HT, van Wees BJ. Energy level alignment at $\mathrm{Co} / \mathrm{AlO}_{\mathrm{x}} /$ pentacene interfaces. J Appl Phys 2007; 101: 093701. 
[106] Shin HJ, Jung MC, Chung J, Kim K, Lee JC, Lee SP. Degradation mechanism of organic light-emitting device investigated by scanning photoelectron microscopy coupled with peel-off technique. Appl Phys Lett 2006; 89: 063503.
[107] For detailed information we want to refer the reader to the Ref. 32$34,40,45,46,66,87,108$

[108] For details of the preparation of the inverted structure $\mathrm{Alq}_{3} / \mathrm{Al}_{2} \mathrm{O}_{3} /$ Co we want to refer the reader to the Ref. 86,106.

(C) Grobosch and Knupfer; Licensee Bentham Open.

This is an open access article licensed under the terms of the Creative Commons Attribution Non-Commercial License (http://creativecommons.org/licenses/ by-nc/3.0/) which permits unrestricted, non-commercial use, distribution and reproduction in any medium, provided the work is properly cited. 